\title{
Swift Gamma-Ray Burst Explorer: Mission Design for Rapid, Accurate Location of Gamma-ray Bursts
}

\author{
David J. Bundas, for the Swift Team \\ Space Missions Branch, Code 594 \\ NASA/Goddard Space Flight Center \\ Greenbelt, MD 20771 \\ 301-286-5573 \\ David.J.Bundas@NASA.gov
}

\begin{abstract}
The Swift Gamma-ray Burst Explorer is a NASA Mid-sized Explorer (MIDEX) with the primary mission of determining the origins of Gamma-Ray Bursts (GRBs). It will be the first mission to autonomously respond to newly-discovered GRBs and provide immediate follow-up with narrow field instruments capable of multi-wavelength (UV, Optical, X-ray) observations. The characteristics of GRBs that are the key mission design drivers, are their non-repeating and brief duration bursts of multi-wavelength photons. In addition, rapid notification of the location and characteristics of the GRBs to ground-and-space-based observatories drive the end-to-end data analysis and distribution requirements.
\end{abstract}

\section{TABLE OF CONTENTS}

1. Overview of the Swift Mission .............................1

2. Description of GRBs ….........................................

3. Driving Requirements ..........................................

4. Requirement Flowdown and Verification ...........3

5. X-ray GRB Position Requirement

Flowdown and Verification ..............................6

6. Burst Alert Message Timing Flowdown

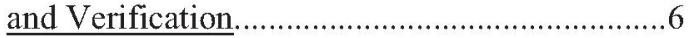

7. Conclusion ……................................................

8. References ……............................................ 7

9. Biography …................................................

\section{OVERVIEW OF THE SWIFT MISSION}

Swift [1] was selected as a NASA MIDEX mission under the Explorer Program in October, 1999. The driving requirements on the mission include:

- Rapid ground notification of newly discovered GRBs (seconds from burst trigger to ground notification),

- Accurate GRB positions (arc minute to sub-arc second), and

- Multi-wavelength observations.

These requirements led to an autonomous observatory that is able to rapidly locate a GRB with the BAT instrument's large field-of-view, and slew the spacecraft to the GRB to bring the narrow field instruments onto the target. This autonomy required an on-orbit decision-making process that was captured in the Figure-of-Merit (FOM) software, which takes input from the instruments and spacecraft, and decides whether a newly discovered GRB warrants an interruption of the current observation. The FOM can then request a slew from the spacecraft, which calculates a safe slew path (avoiding Sun, Moon, and Earth), and rapidly re-points the observatory.

The GRB location accuracy requirements drove instrument and spacecraft design, as well as the design of the Optical Bench (OB), which maintains the co-alignment of the three instruments with respect to the attitude control system reference.

\section{Organization}

The Swift mission is managed by the GSFC, and includes an international team with key contributions from the UK and Italy. The spacecraft bus, provided by General Dynamics C4 Systems (formerly Spectrum Astro, Inc.) was procured through a Rapid Spacecraft Development Office (RSDO) contract by the GSFC. There are three instruments: the Burst Alert Telescope (BAT) provided by the GSFC; the X-Ray Telescope (XRT) provided by a team led by the Pennsylvania State University (PSU); and the Ultra-Violet/Optical Telescope (UVOT), again managed by PSU. The Mission Operations Center (MOC) was developed by Omitron, Inc. under the direction of PSU. Science archiving and data analysis centers are located at the GSFC, in the UK and in Italy.

\section{Observatory Overview}

The Swift observatory consists of a spacecraft bus and a payload module, which includes 3 instruments mounted on a high stability optical bench. See Figure 1.0(a). The BAT instrument monitors $1 / 6$ of the sky for GRBs, while the narrow field XRT and UVOT instruments carry out planned science observations. When BAT detects a burst the spacecraft slews the narrow field instruments (NFIs) onto the burst, so that they can collect data on the GRB afterglow immediately (within $\sim 1$ minute) after the burst. This rapid response is one of the key features of the Swift observatory, which will greatly enhance the information available on GRBs.

\section{Spacecraft Bus}

The Swift S/C bus was based on Spectrum Astro's NASA RSDO Catalog SA-200 S/C series and provides a highly accurate and maneuverable platform for science observations.

The spacecraft bus consists of an 8 sided structure with electronics boxes mounted to the exterior. The interior of the structure is left open to accommodate the two NFIs. The payload module is mated to the spacecraft bus through 3 titanium flexures, providing a relatively stress free interface to the instrument module. Power is provided by two solar array wings which generate 2100 Watts, and an $80 \mathrm{~A}-\mathrm{hr} \mathrm{NiH} 2$ battery. Power is distributed unregulated to the payload. Omni-directional antenna coverage is provided by 2 anten- 


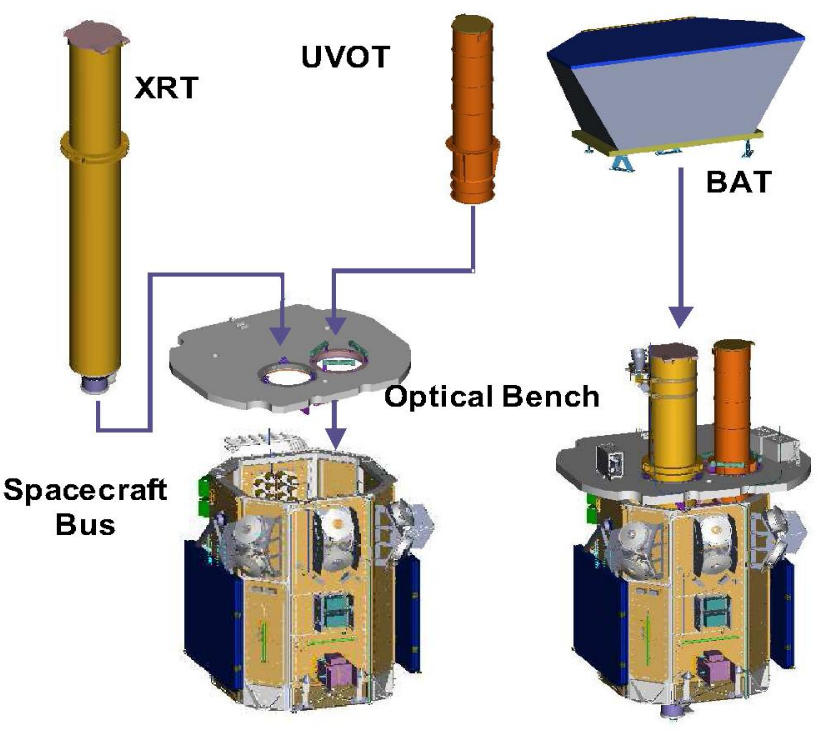

Figure 1.0(a): The Swift observatory was designed to allow parallel development paths for the spacecraft bus and each instrument.

nas located on the instrument module sunshade, and 2 antennas mounted to deployable booms at the aft end of the spacecraft. Science data are stored during the orbit and downloaded over the ground station at Malindi, Kenya (2.2 Mbps). The burst alert messages as well as spacecraft emergency messages are relayed through the TDRSS Demand Access System (DAS), which allows continuous coverage for spacecraft events.

The very capable attitude control system meets the demands for rapid response to the discovery of new bursts by providing rapid slewing and settling performance. It also includes autonomous planning software to avoid bright sources while slewing to the new target. The primary actuators are six reaction wheels mounted in a hexagonal configuration, each capable of delivering $0.68 \mathrm{~N}-\mathrm{m}$ of torque.

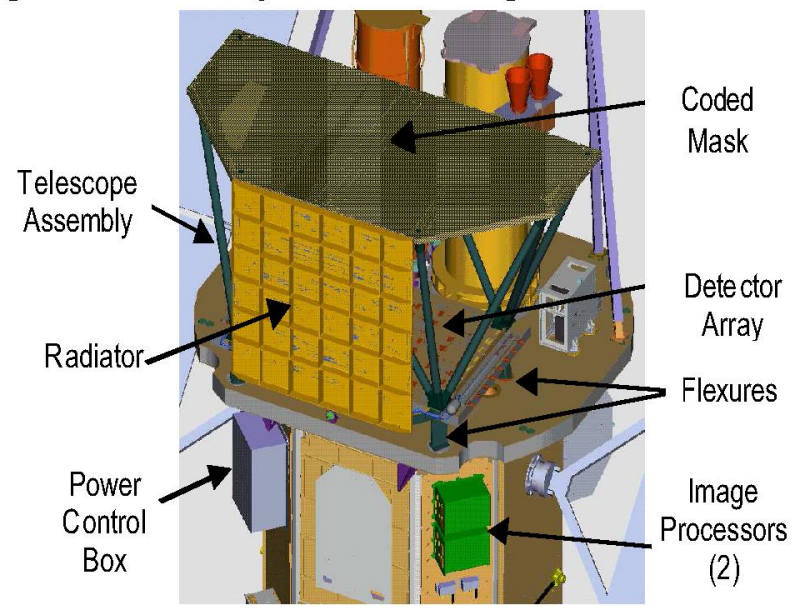

Figure 1.0(b): The BAT telescope assembly shown mounted on the spacecraft bus.

\section{Burst Alert Telescope (BAT)}

The BAT [2] is a coded mask imager with a detector array comprised of 32,768 individual Cadmium-Zinc-Telluride detectors, each with a separate measurement chain. See Figure $1.0(\mathrm{~b})$. Imaging is enabled by providing a mask of lead tiles forming a pseudo-random pattern in the field-of-view of the detectors. When the detector counts exceed a threshold level, image processing on board determines the burst location by a Fourier transform imaging technique. Key instrument parameters are listed in Table 1.0(a).

Table 1.0(a): BAT parameters

\begin{tabular}{|l|}
\hline Aperture: Coded Mask \\
\hline Detecting Area: $5200 \mathrm{~cm}^{2}$ \\
\hline Detector: CdZnTe \\
\hline Detector Operation: Photon Counting \\
\hline Field of View: $2.0 \mathrm{sr}$ (partially coded) \\
\hline Detection Elements: 256 modules of 128 elements \\
\hline Detector Size: $4 \mathrm{~mm}$ x $4 \mathrm{~mm}$ x $2 \mathrm{~mm}$ \\
\hline Telescope PSF: 17 arcminutes \\
\hline Location Accuracy: $1-4$ arcminutes \\
\hline Energy Range: $15-150 \mathrm{keV}$ \\
\hline
\end{tabular}

\section{$X$-ray Telescope $(X R T)$}

The XRT [3] is a $3.5 \mathrm{~m}$ focal length Wolter-type X-ray telescope capable of centroiding to about an arc second over the range 0.2 to $10 \mathrm{keV}$. See Figure $1.0(\mathrm{c})$. A list of instrument parameters is given in Table 1.0(b). Managed by PSU, the instrument is a collaboration between PSU, University of Leicester (UL), and the Osservatorio Astronomico di Brera (OAB), Milano, Italy. UL was responsible for the focal plane camera assembly, and $\mathrm{OAB}$ provided the nested grazing incidence mirrors.

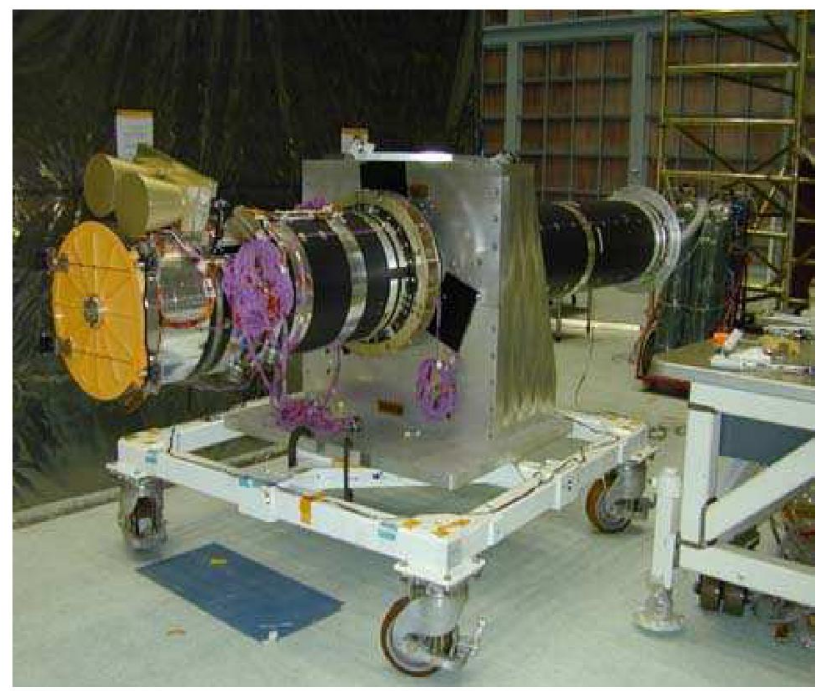

Figure 1.0(c): The XRT instrument shown during I\&T at the GSFC. Note the Star Tracker simulators attached to the forward tube.

\section{Ultra-Violet/Optical Telescope (UVOT)}

The UVOT provides the highest resolution images of the GRB afterglow. It is based on the XMM/OM instrument, and consists of a $30 \mathrm{~cm}$ aperture, Ritchey-Chretien optical system, a six-element filter wheel, and an image intensified CCD. See Figure 1.0(d). The system is capable of detecting 
objects as dim as magnitude 24 . Internal stability is provided by an iso-thermal design that provides a constant environment for the heater controlled optical metering structure. A listing of key instrument parameters is given in Table 1.0(c). UVOT was managed by PSU, and the telescope module was built by Mullard Space Science Laboratory (MSSL) of University College, London in the UK.

Table 1.0(b): XRT Parameters

\begin{tabular}{|l|}
\hline X-Ray Telescope \\
\hline Telescope: Wolter I \\
\hline Detector: XMM EPIC CCD \\
\hline Effective Area: $135 \mathrm{~cm}^{2}$ at $1.5 \mathrm{keV}$ \\
\hline $\begin{array}{l}\text { Detector Operation: Photon Counting, Integrrated Imag- } \\
\text { ing, and Rapid Timing }\end{array}$ \\
\hline Field of View: 23.6 x 23.6 arcminutes \\
\hline Detection Element: $600 \times 600$ pixels \\
\hline Pixel Scale: 2.36 arcsec/pixel \\
\hline Telescope PSF: 18 arcsec HPD at $1.5 \mathrm{keV}$ \\
\hline Location Accuracy: $3-5$ arcseconds \\
\hline Energy Range: $0.2-10 \mathrm{keV}$ \\
\hline Sensitivity: $2 \times 10-14$ ergs $\mathrm{cm}^{2}{ }^{2} \mathrm{~s}^{-1}$ in $10^{4} \mathrm{sec}$ \\
\hline
\end{tabular}

\section{DESCRIPTION OF GRBS}

GRBs were detected by the Vela4 satellite in 1967, although the announcement of their discovery did not occur until 1973. With this discovery, some of the basic characteristics of GRBs were revealed. First was their short-lived nature, producing gamma-ray flashes that last for only seconds before decaying into the background. Another characteristic is their random distribution in the sky, indicating great distances and extremely powerful explosions. In recent years it has been discovered that the brief, initial burst of gamma rays is followed by a multi-wavelength afterglow that may be detectable for weeks afterward.

\section{DRIVING REQUIREMENTS}

The Swift challenge of studying these GRBs is to provide for the rapid detection and localization of the GRB, followed up
Table 1.0(c): UVOT Parameters

\begin{tabular}{|l|}
\hline Ultraviolet/Optical Telescope \\
\hline Telescope: Modified Ritchey-Chretien \\
\hline Aperture: $30 \mathrm{~cm}$ diameter \\
\hline F-number: 12.7 \\
\hline Detector: Intesified CCD \\
\hline Detector Operation: Photon Counting \\
\hline Field of View: $17 \times 17$ arcminutes \\
\hline Detection Element: $2048 \times 2048$ pixels \\
\hline Telescope PSF: 0.9 arcsec at $350 \mathrm{~nm}$ \\
\hline Location Accuracy: 0.3 arcseconds \\
\hline Wavelength Range: $170 \mathrm{~nm}-650 \mathrm{~nm}$ \\
\hline Colors: 6 \\
\hline Spectral Resolution (Grisms): $\lambda / \Delta \lambda \sim 200$ at $400 \mathrm{~nm}$ \\
\hline Sensitivity: $B=24$ in white light in $1000 \mathrm{sec}$ \\
\hline Pixel Scale: 0.48 arcseconds \\
\hline Bright Limit: mv = 7 mag \\
\hline
\end{tabular}

with multi-wavelength observations of the afterglow. Figure 3.0(a) describes the flowdown of key science objectives into system requirements. The driving requirements on the system design are to:

- Report coarse GRB position within 10 arcmin in 30 seconds

- Report X-ray GRB position within 5 arc seconds within 100 seconds

- Report UV/Optical position within 0.3 arc seconds within 270 seconds

\section{REQUIREMENT FLOWDOWN AND VERIFICATION}

The initial burst detection by BAT must be accurate enough to allow the burst to fall within the field of view of the NFIs after a slew of up to 100 degrees. The coarse GRB position requirement error budget is shown in Figure 3.0(a). The first tier of the error budget covers BAT internal alignment stability and centroiding capability, as well as spacecraft alignment stability and attitude knowledge accuracy. BAT

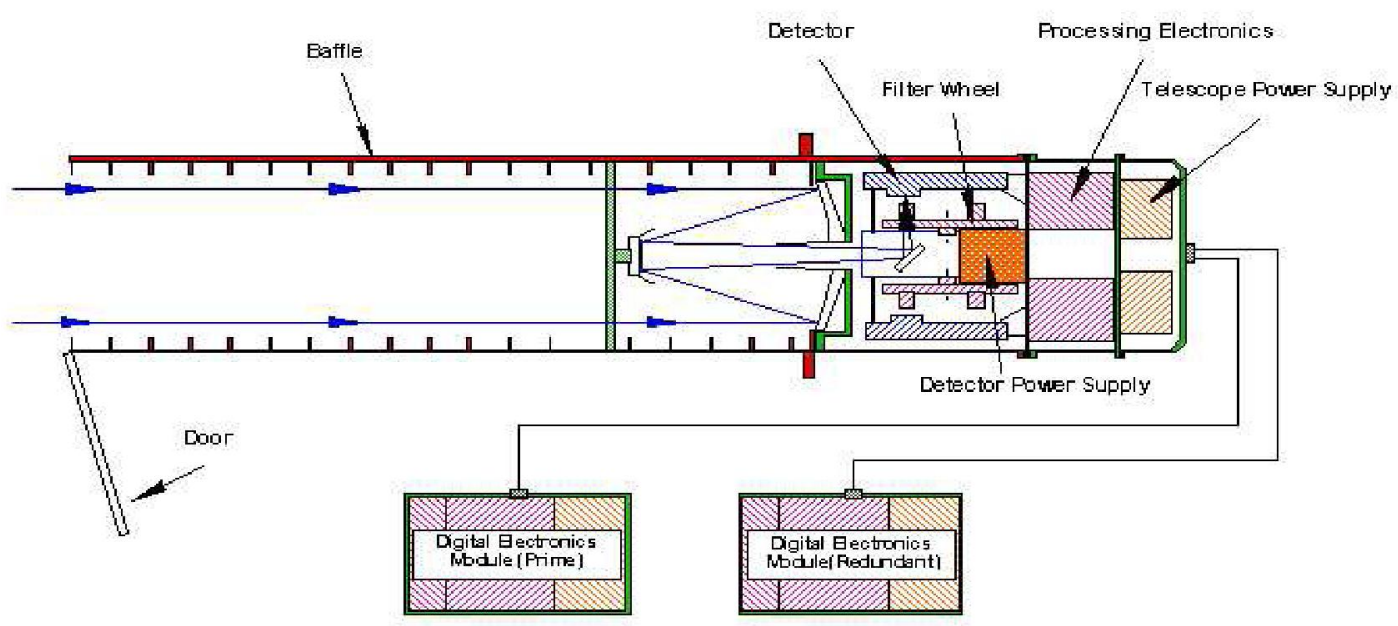

Figure 1.0(d): UVOT block diagram 
internal alignment stability was verified by a combination of tests and analyses, and was within the allocations. Figure 4.0(a) gives the original allocation as well as the predictions for on-orbit performance. Vibration and thermal testing veri- fied the ability of the BAT structure to survive the launch and orbital environments without suffering alignment shifts.

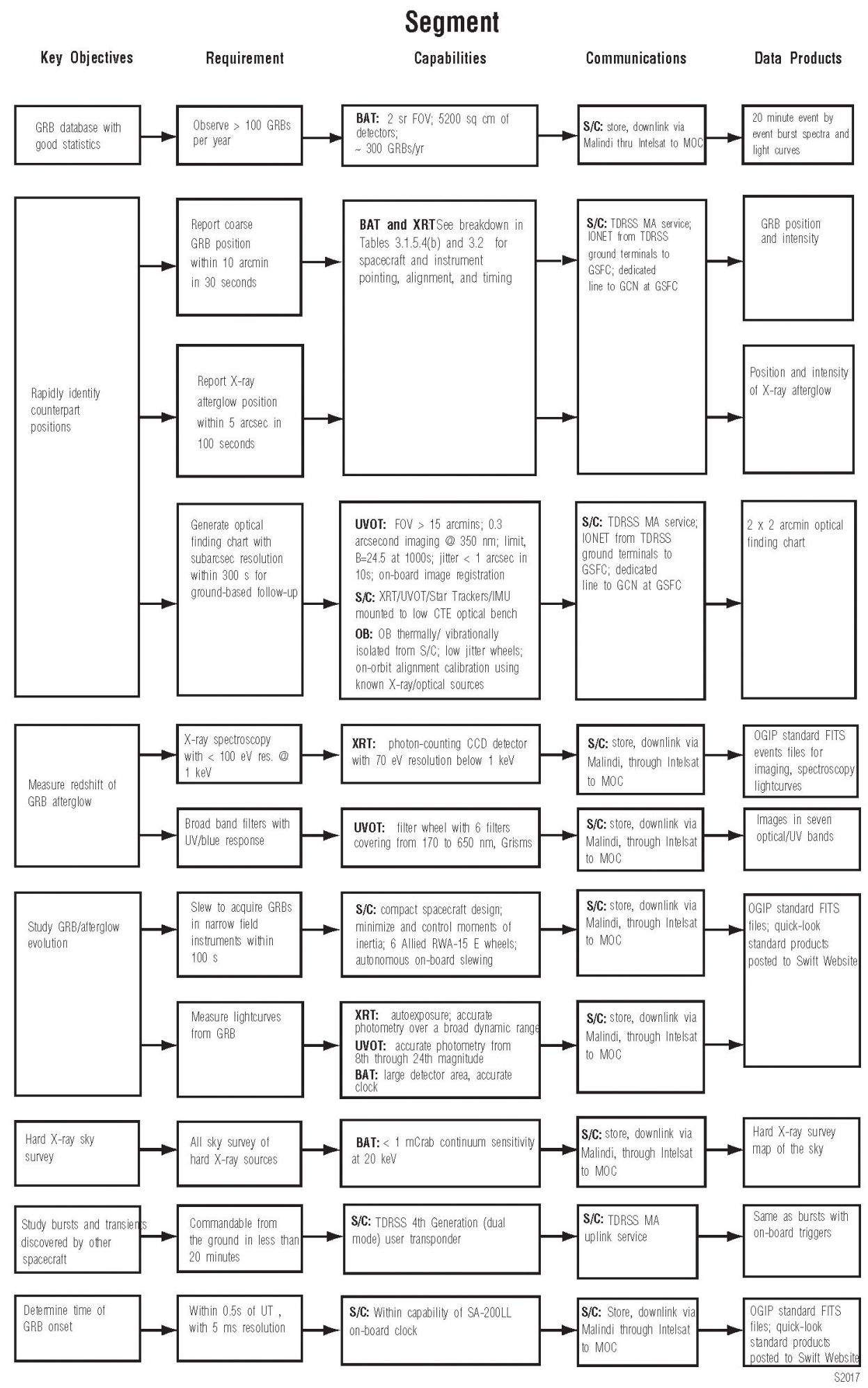

Figure 3.0(a): GRB MilDEX 


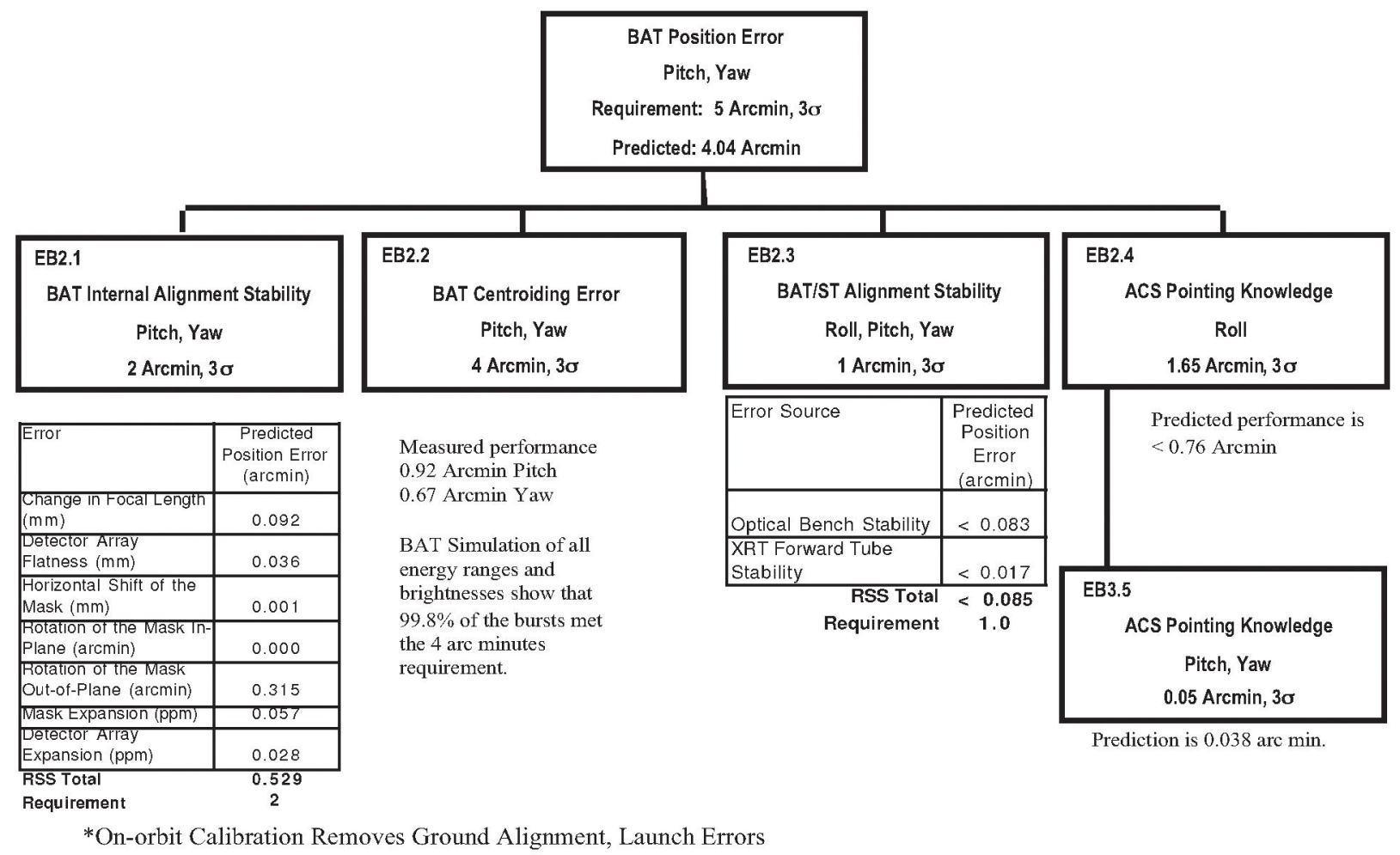

Figure 4.0(a): BAT GRB Position Error Budget

One time alignment shifts due to launch and ground handling events will be calibrated out of the system on-orbit. This was backed up with a structural/thermal analysis that showed alignment was maintained through the predicted orbital thermal environments. The BAT centroiding error was both tested and simulated to verify that it meets requirements. A radioactive source was suspended above the BAT instrument and the resulting image's point spread function was determined to be less than an arc minute. A complete simulation of the BAT instrument was developed to provide a way to test the software that converts detector counts into images of the sky. This simulation was run with a 1000 simulated bursts covering the expected intensities and energy distributions expected on orbit. The results showed that 998 bursts were located to within 4 arc minutes. More than half of the simulated bursts were located to within 1.2 arc minutes accuracy. ACS accuracy and platform stability requirements are driven by the much tighter NFI requirements, and therefore, easily meet the coarse GRB position error budget allocations.

\section{X-RAY GRB Position REQUiREMENT FLOWDOWN AND VERIFICATION.}

The XRT must return a GRB position that is accurate to within 5 arc seconds for follow-up by ground observers. The requirement flows into allocations for XRT centroiding error, XRT and platform mechanical stability, and ACS pointing accuracy, as shown in Figure 5.0(a). The XRT design includes an active thermal control system and a low CTE graphic epoxy tube that forms the metering structure between the grazing incidence mirror and the cooled CCD. XRT Centroiding error was evaluated during instrument calibration testing at the Panter X-ray beam facility in Germany, in the fall of 2003.

The spacecraft ACS system provides accurate attitude knowledge to the instruments once settled on target. On-orbit boresight calibration between the XRT and the Star
Trackers will make use of the most benign on-orbit conditions, to remove all one-time launch shift effects.

An additional feature of the XRT system design is the telescope alignment monitor, which can measure the misalignment between the focal plane, the instrument interface, and the star tracker baseplate. The last element of the error budget was introduced because the design of the telescope includes a heat pipe, harnessing, and thermal blanket closeouts that could affect pointing. These items introduce loads into the unsupported aft end of the telescope, and each one was treated carefully to ensure minimal impact. The heat pipe design included flexure mounts that prevent lateral loading. A mockup of the flight harness was used to test its compliance to relative motion between the telescope and the harness support plate on the spacecraft. Thermal blankets were carefully closed out by overlapping sections such that the blankets could not constrain motion of the telescope. The final results of the pointing performance will require the completion of on-orbit calibration between the boresight of XRT and the star trackers. This is planned during the early orbit checkout period.

\section{Burst Alert Message Timing FLOWDOWN AND VERIFICATION.}

The rapid notification of the science community upon discovery of new GRBs is a critical science requirement. The BAT, XRT, and UVOT GRB position notices must be available at the Gamma ray burst Coordinates Network (GCN) within 30,100, and 270 seconds, respectively of the initial BAT-detected GRB. What distinguishes Swift from other observatories is its ability to autonomously re-target based on in-orbit discoveries. Current missions take hours or days to re-point, much too long to capture the dying GRB afterglow. Table 6.0(a) gives the allocation of these timing requirements to the various Swift elements. Across the top of 


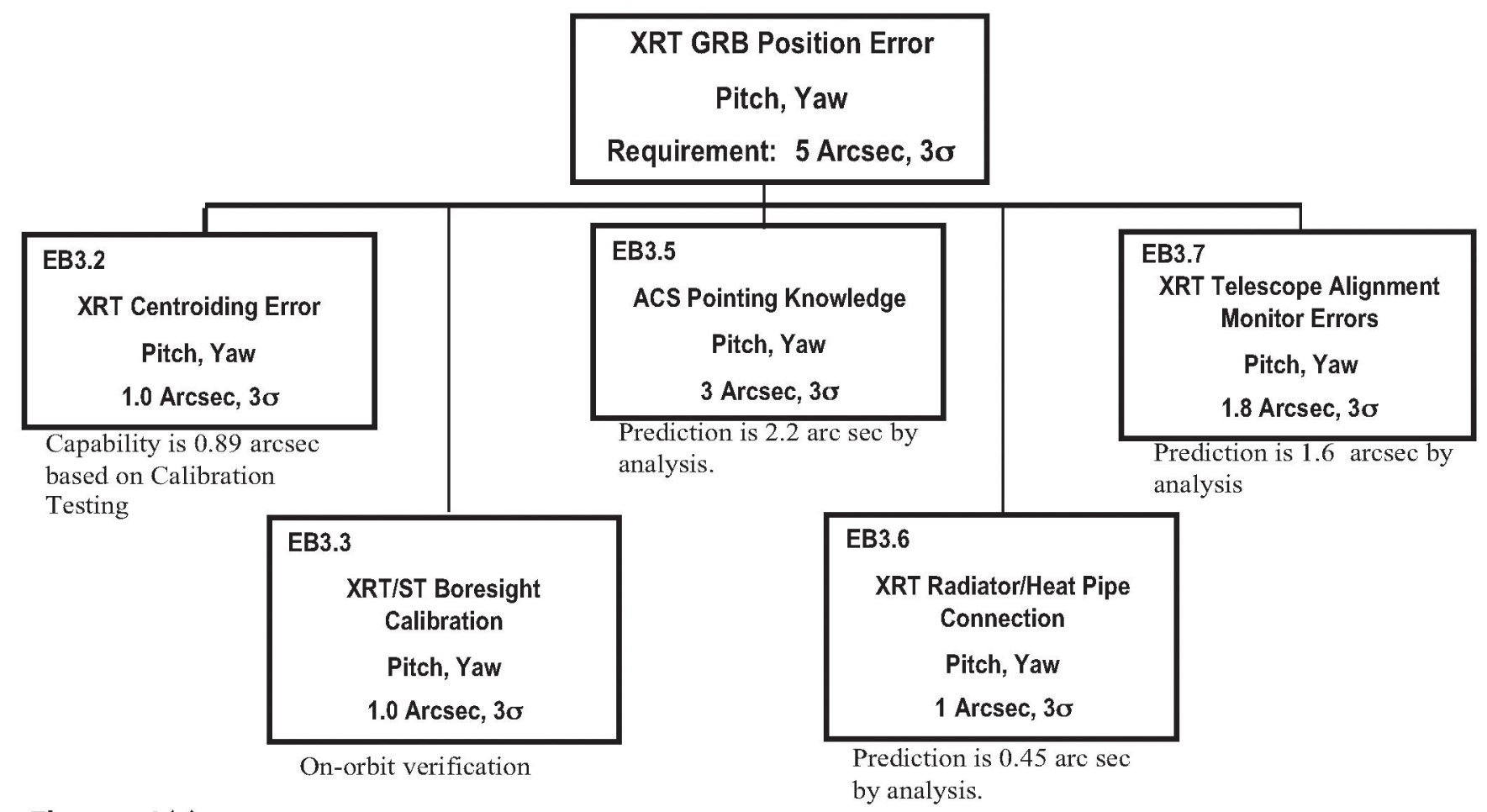

Figure 5.0(a): XRT GRB Position Error Budget

the table are the burst alert messages that are transmitted by Swift on discovery of a GRB. The rows of the table correspond to the individual delays in getting the burst alert message to the ground. These include instrument and FOM processing time, spacecraft planning and slewing time, and communications delays. Each of the time requirements starts with the burst being detected by the BAT instrument, and

Table 6.0(a): Message Timing Budget ends with a message leaving the GCN notifying other ground and orbiting observatories that a GRB has been discovered. A breakdown of the timing requirements is presented in Table 6.0(a), showing how each of the steps in the GRB detection and notification process was broken down to the level where it could be allocated to a single mission element.

\begin{tabular}{|c|c|c|c|c|c|c|c|c|c|c|}
\hline SRD source & & SRD2.1 & & & SRD2.3 & & SRD2.5 & & & \\
\hline Data Product $\Rightarrow$ & GRB Alert & $\begin{array}{c}\text { BAT } \\
\text { Position }\end{array}$ & $\begin{array}{c}\text { Fon } \\
\text { Will/Will } \\
\text { Not } \\
\text { Observe }\end{array}$ & $\begin{array}{c}\text { S/C } \\
\text { Will/Will } \\
\text { Not } \\
\text { Observe }\end{array}$ & $\begin{array}{c}\text { XRT } \\
\text { Position }\end{array}$ & $\begin{array}{l}\text { BATLight- } \\
\text { curve }\end{array}$ & $\begin{array}{l}\text { UvoT } \\
\text { Finding } \\
\text { Chart }\end{array}$ & $\begin{array}{c}\text { XKT } \\
\text { Spectrum }\end{array}$ & $\begin{array}{l}\text { XRT } \\
\text { Image }\end{array}$ & Howdown \\
\hline Message Size (In Bytes) & $\leq 58$ & $s 92$ & $\leq 68$ & $\leq 68$ & $\leq 60$ & $\leq 2150$ & $\leq 2000$ & $\leq 2200$ & $\leq 680$ & Instruments \\
\hline Estimated No. of Telemetry Packets & 1 & 1 & 1 & 1 & 1 & 3 & 3 & 3 & 1 & (info only) \\
\hline Estimated No. of Telemetry Frames & 1 & 1 & 1 & 1 & 1 & 21 & 19 & 21 & 7 & (info only) \\
\hline BAT GRB Location/ FoM Slew Request (s) & & 6.0 & 6.2 & 6.2 & 6.2 & 6.2 & 6.2 & 6.2 & 6.2 & BAT/foM \\
\hline $\begin{array}{l}\text { BATYFoM to C\&DH, ACS Slew Check \& Request to } \\
\text { Safe UVOT (s) }\end{array}$ & 0.4 & 0.4 & 0.4 & 0.8 & 1.0 & 1.0 & 1.0 & 1.0 & 1.0 & $\mathrm{~s} / \mathrm{C}$ \\
\hline UVOT Safing (s) & & & & & 1.0 & 1.0 & 1.0 & 1.0 & 1.0 & UVOT \\
\hline UVOT Reply to C\&DH (s) & & & & & 0.4 & 0.4 & 0.4 & 0.4 & 0.4 & $\mathrm{~s} / \mathrm{C}$ \\
\hline Spacecraft slew/settle (s) & & & & & 75.0 & & 75.0 & 75.0 & 75.0 & $\mathrm{~s} / \mathrm{C}$ \\
\hline Instrument to C\&DH (s) & & & & & 0.3 & 0.5 & 1.4 & 0.8 & 0.4 & $8 / C$ \\
\hline C\&DH to Transponder (s) & 0.4 & 0.4 & 0.4 & 0.4 & 0.4 & 0.8 & 0.8 & 0.8 & 0.5 & $\mathrm{~S} / \mathrm{C}$ \\
\hline S/C to TDRSS Initial Delay (s) & 9.0 & 4.0 & 3.0 & 4.0 & 0.0 & 0.0 & 0.0 & 0.0 & 0.0 & $8 / C$ \\
\hline Swift to WSC @1 kbps (s) & 2.3 & 2.3 & 2.3 & 2.3 & $2 . \hat{3}$ & 22.8 & 20.8 & 22.8 & 8.5 & $8 / C$ \\
\hline WSC processing (s) & 3 & 3 & 3 & 3 & 3 & 3 & 3 & 3 & 3 & Ground Systems \\
\hline WSC to $\mathrm{GCN}(\mathrm{s})$ & 0.3 & 0.3 & 0.3 & 0.3 & $0 . \hat{3}$ & 0.3 & 0.3 & 0.3 & 0.3 & Ground Systems \\
\hline GCN Processing (s) & 0.1 & 0.1 & 0.1 & 0.1 & 0.1 & 0.2 & 0.5 & 0.5 & 0.5 & $\mathrm{GCN}$ \\
\hline Allocated Total Times (s) & 15.5 & 16.5 & 17.7 & 19.1 & 95.0 & 151.2 & 260.4 & 311.8 & 103.8 & \\
\hline End-to-End Timing Requirement (s) & 20 & 20 & 70 & 70 & 100 & 200 & 270 & 1200 & 1200 & \\
\hline Margin (Time) & 4.5 & 3.5 & 52.3 & 50.9 & 5.0 & 48.8 & 9.6 & 888.2 & 1096.2 & \\
\hline Margin $(\%)$ & $29 \%$ & $21 \%$ & $295 \%$ & $266 \%$ & $5 \%$ & $32 \%$ & $4 \%$ & $285 \%$ & $1056 \%$ & \\
\hline
\end{tabular}


To achieve these rapid X-ray and UV/Optical afterglow observations requires a highly autonomous observatory that must evaluate whether a burst is scientifically interesting; plan a slew which avoids the Earth, Sun, and Moon; rapidly and accurately settle on the newly-discovered burst; and provide the ground with information on the bursts so that follow-up observations are possible. Key aspects of the autonomous design were its simple instrument/spacecraft interfaces and the on-board slew planning.

The simple interface allows messaging and calculations to be kept to a minimum, and permits subsystem level testing to verify interfaces. The other key to the design is that the spacecraft is responsible for maintaining the safety of the observatory. The NFIs could be damaged by slews across bright objects, so this drove the requirement for on-board bright object avoidance during slews. Several issues were resolved satisfactorily during the development process, including slew speed limitations to give enough time to recover from a $\mathrm{C} \& D H$ reboot, impact of mass growth on slew speed, and added slew time to account for additional roll for slews when crossing the ecliptic plane.

\section{Conclusions}

The driving requirements and design of the Swift mission have been described. The flowdown of requirements into the elements of the mission through the pointing and message timing error budgets defined the challenge for the team. The end-to-end verification process employed gives the team confidence that the mission will meet its requirements and science objectives as defined at the outset of the program.

\section{REFERENCES}

[1] Neil Gehrels, "The Swift $\gamma$-ray burst mission", New Astronomy Review, v48:431-435, 2004 April

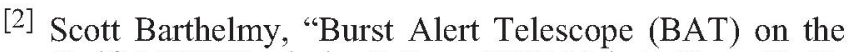
Swift MIDEX mission", Proc. SPIE Vol. 4140, p. 50-63, $\mathrm{X}$-Ray and Gamma-Ray Instrumentation for Astronomy XI, Kathryn A. Flanagan; Oswald H. Siegmund; Eds., 2000 December

${ }^{[3]}$ D. N. Burrows, "The Swift X-ray Telescope: Catching X-ray afterglows of GRBs on the fly!", American Astronomical Society Meeting, v202, 2003 May

${ }^{[4]}$ P. W. A. Roming, S. D. Hunsberger, J. A. Nousek, K. O. Mason, and A. A. Breeveld, "The Swift Ultra-Violet/Optical Telescope", American Astronomical Society Meeting, v202, 2003 May

[5] John C. Ong and Mike Rackley, "AUTONOMOUS OPERATIONS FOR THE SWIFT MISSION", SpaceOps 2002 in Houston, TX, October 9-12, 2002

\section{BIOGRAPHY}

David Bundas has served as the Swift Systems Manager since January 1999. He has provided systems analysis and engineering expertise to a variety of space and earth science missions since joining the NASA/GFSC in 1983, after receiving his B.S. and M.S. degrees in Aeronautics and Astronautics from M.I.T. 